\title{
Metastatic Renal Cell Carcinoma to the Sinonasal Cavity: A Case Series
}

\author{
Aaron K. Remenschneider ${ }^{1}$ Peter M. Sadow ${ }^{2}$ Derrick T. Lin ${ }^{1} \quad$ Stacey T. Gray ${ }^{1}$ \\ ${ }^{1}$ Department of Otology and Laryngology, Massachusetts Eye and Ear \\ Infirmary, Harvard Medical School, Boston, Massachusetts, United States \\ 2 Department of Pathology, Massachusetts General Hospital, Harvard \\ Address for correspondence Aaron Remenschneider, MD, 243 Charles \\ Street, Boston, MA 02114, United States \\ (e-mail: aaron_remenschneider@meei.harvard.edu).
}

Medical School, Boston, Massachusetts, United States

J Neurol Surg Rep 2013;74:67-72.

\begin{abstract}
Objectives To describe the presentation, work-up, and management of patients with metastatic renal cell carcinoma (RCC) to the sinonasal cavity and skull base, and to describe our current treatment algorithm of endoscopic surgical resection followed by radiation therapy.

Design Retrospective review of two recent cases from our institution over a 1-year period, with a relevant review of the literature.

Setting A large regional tertiary care facility.

Participants Consecutive cases of RCC with metastases to the sinonasal cavity presenting to our institution.

Main Outcome Measures Preoperative and postoperative sinonasal outcome test (SNOT)-22 scores, duration of hospital stay, complications, and local disease control

Results Patients in this series underwent preoperative embolization followed by endoscopic resection without complication. Postoperatively they were treated with

Keywords

- renal cell carcinoma

- quality of life

- embolization

- epistaxis

- nasal obstruction radiation therapy. They experienced improvement in their SNOT-22 scores and are currently free of local disease.

Conclusion Metastatic RCC to the sinonasal cavity can be safely treated with preoperative embolization followed by endoscopic surgical resection and radiation therapy, which can result in improvement in sinonasal quality of life and is a potential adjunct for local control of disease.
\end{abstract}

\section{Background}

Renal cell carcinoma (RCC) is a relatively rare tumor, accounting for just over $2 \%$ of all adult malignancies as of $2008 .{ }^{1}$ Metastatic disease occurs through hematogenous spread that most often manifests in bone, lung, and the liver; however, the head and neck have been reported sites of metastasis in up to $15 \%$ of patients. ${ }^{2}$ Metastatic disease tends to present at or near the time of initial diagnosis, but there have been reported cases of metastases to the head and neck manifesting greater than 10 years following primary nephrectomy. ${ }^{2,3}$

received

May 10, 2012 accepted after revision

March 10, 2013

published online

May 9, 2013
The most common site of metastatic disease in the head and neck is the thyroid gland, but the sinonasal cavity is also a frequent site, and metastases to the neck, tongue, ${ }^{4}$ facial skin, and other sites have also been reported. ${ }^{2,5,6}$ Epistaxis, facial pain, or nasal obstruction may be the presenting symptom for patients with metastatic RCC. ${ }^{7,8}$ Tumor in the sinonasal cavity can result in a significant decrease in quality of life due to recurrent severe epistaxis, nasal obstruction, and local pain. ${ }^{9}$ Any patient presenting with nasal obstruction or epistaxis and a history of RCC should have metastatic disease included in the differential diagnosis.
License terms

Stuttgart · New York

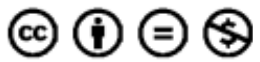

10.1055/s-0033-1346972. ISSN 2193-6358. 


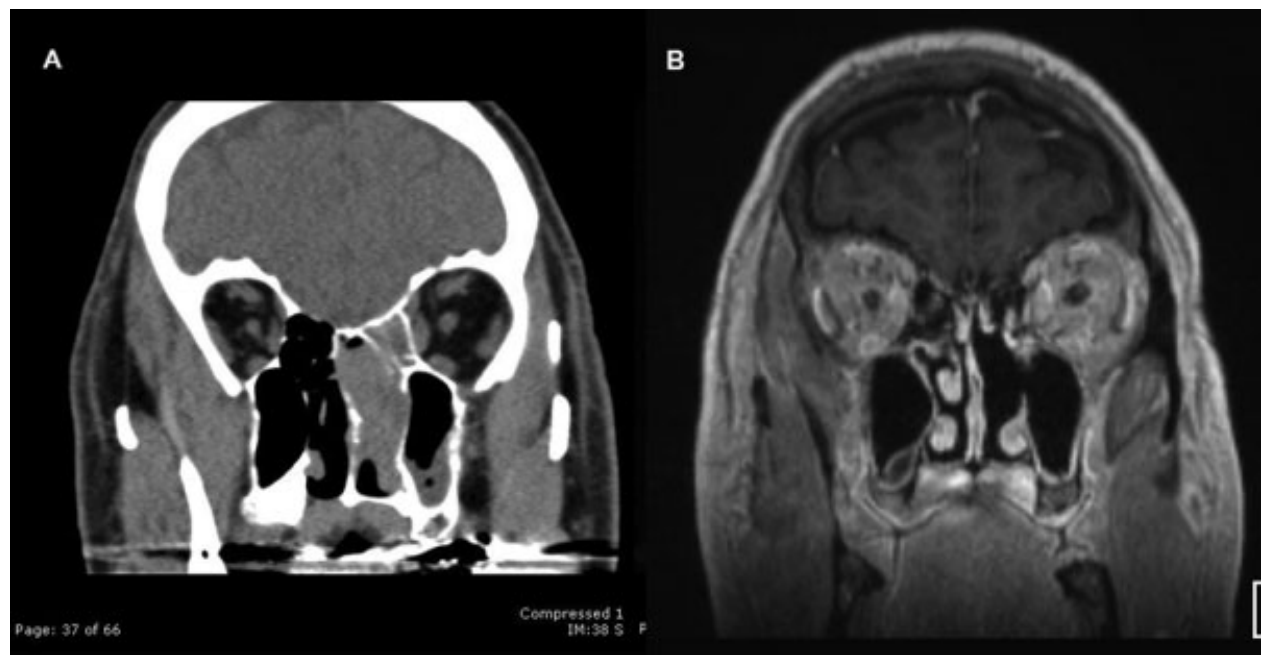

Fig. 1 (A) Noncontrast coronal computed tomography scan demonstrating soft tissue mass extending to the cribriform plate and skull base. (B) 18-month postoperative $\mathrm{T} 1$ postcontrast magnetic resonance imaging demonstrating patent left maxillary antrostomy and no evidence of recurrent disease.

The typical work-up for a patient with a presumed sinonasal mass should include nasal endoscopy, followed by prebiopsy imaging with contrast-enhanced computed tomography (CT) and magnetic resonance imaging (MRI) if the lesion extends to the skull base. Several reports have described profuse bleeding at the time of biopsy of sinonasal RCC due to the hypervascular nature of these tumors, requiring external carotid ligation; ${ }^{10}$ thus, any biopsy of a suspicious lesion should be performed in the operating room. Historically, external beam radiation therapy has been the treatment of choice for metastatic sinonasal RCC, with or without concurrent chemotherapy. ${ }^{10}$ Surgical resection has been controversial and usually reserved for small solitary lesions or for debulking after primary radiotherapy.

We present two cases of metastatic RCC to the sinonasal cavity and skull base that were successfully resected endoscopically after preoperative embolization. Both patients had significant sinonasal symptoms that resolved posttreatment. We discuss each patient's presentation, detail our institution's work-up and treatment algorithm, and highlight the safety and minimal impact of surgery followed by postoperative radiation.

\section{Case 1}

A 53-year-old white man had a 4-month history of left-sided nasal obstruction and facial pressure. He noted discolored, brown nasal drainage, but did not have frank epistaxis. At the time of initial evaluation his sinonasal outcome test (SNOT22) score was 32 (where 7 or less is "normal"11 and higher values indicate more severe sinonasal symptoms). His past medical history was significant for a right nephrectomy for a T1N0 renal cell carcinoma 10 years prior to presentation. On exam, nasal endoscopy was remarkable for a mass in the left nasal cavity, medial to the middle turbinate. A CT scan revealed a vascular, expansile, polypoid soft tissue mass, measuring $4 \times 3 \times 2 \mathrm{~cm}$ and filling the space medial to the middle turbinate and the sphenoethmoid recess, extending into the sphenoid sinus and nasopharynx. Superiorly, it extended to the cribriform plate but did not appear to breach the skull base, and bony remodeling along the medial maxillary wall was noted. There was no radiologic evidence of intracranial extension (-Fig. 1).

An initial biopsy resulted in extensive bleeding, and pathology was suggestive of a benign process, such as hemangioma. Subsequently he was referred to our service for further treatment. Given the degree of bleeding at the time of initial biopsy, preoperative embolization was performed. The angiography at the time of embolization showed that the majority of the blood supply was from the internal carotid system via the anterior ethmoid arteries, which could not be embolized

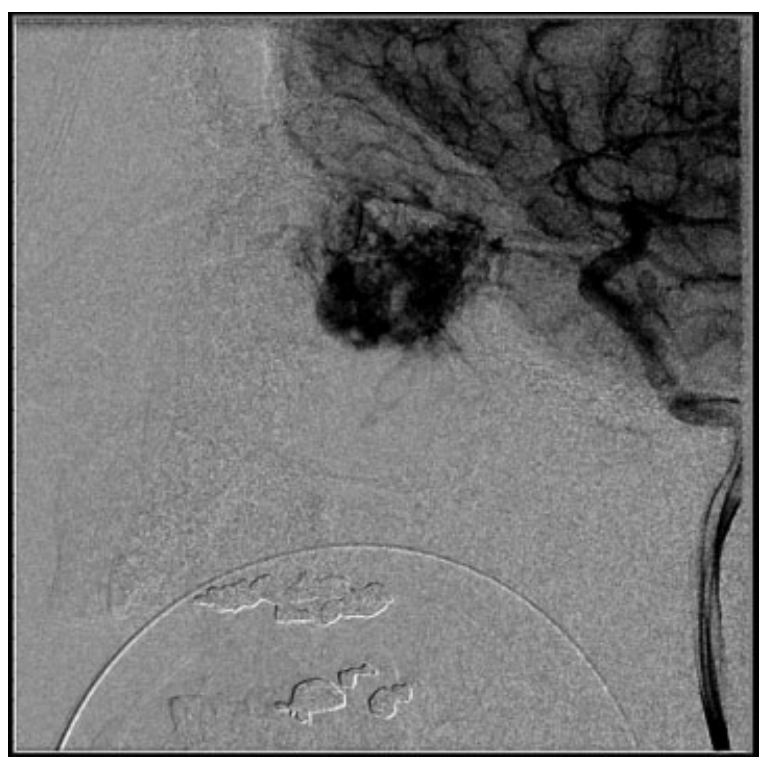

Fig. 2 Sagittal angiogram of the left internal carotid demonstrating significant anterior ethmoid artery contribution to the lesion. 


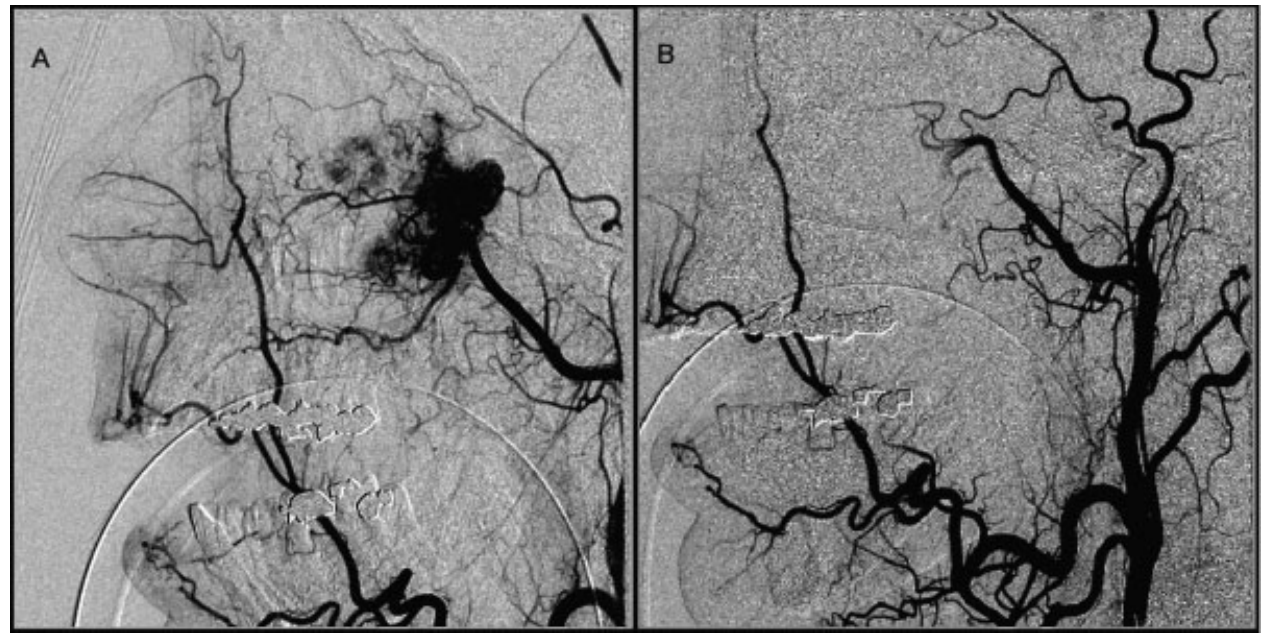

Fig. 3 (A) Sagittal angiogram of the left external carotid showing sphenopalatine contribution. (B) Postembolization of the left sphenopalatine artery.

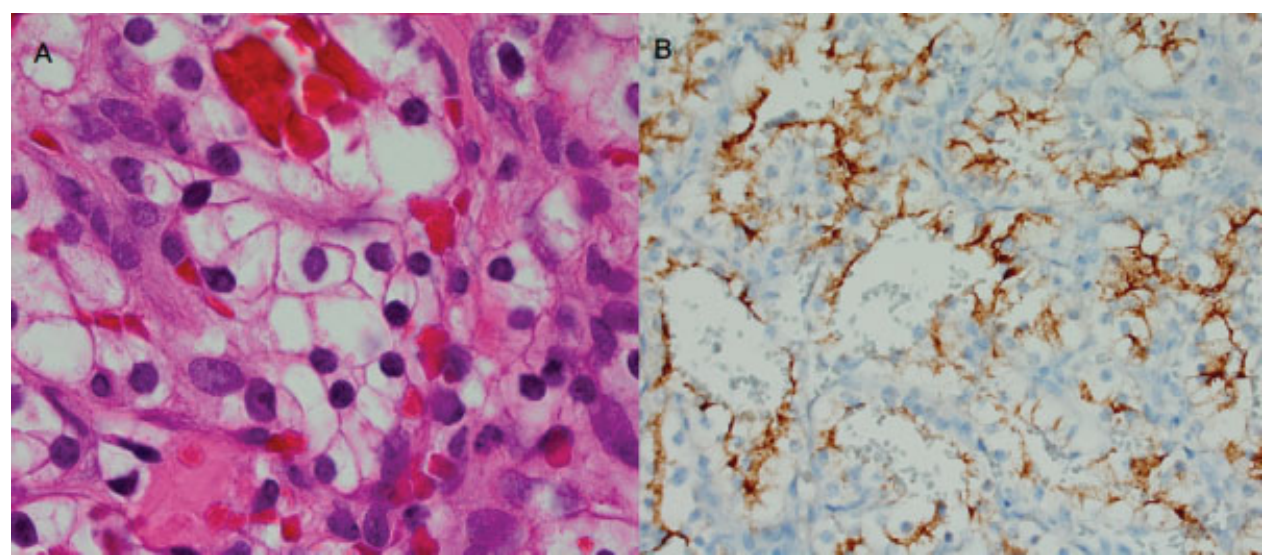

Fig. 4 (A) A 1000× hematoxylin and eosin stain showing typical clear cells arranged in nests with pooling of red blood cells. (B) Renal cell carcinoma antigen confirms the diagnosis with apical cytoplasmic staining.

(-Fig. 2). Contributions from the sphenopalatine artery were embolized (-Fig. 3).

The day following embolization, the mass was removed endoscopically en bloc with minimal intraoperative bleeding after selective endoscopic bipolar cautery of several feeding vessels from the anterior ethmoid artery. The patient tolerated the procedure well and was discharged the day following surgery without complication. Final pathology was consistent with RCC and demonstrated typical clear cells arranged in nests that stained positively for RCC antigen ( $\mathbf{F i g . 4}$ ).

The patient received postoperative radiation therapy. Twelve months after completion of treatment, he was found to have a new thyroid mass that was suspicious for papillary thyroid carcinoma on fine-needle aspiration. He underwent total thyroidectomy, which confirmed papillary thyroid carcinoma but was also incidentally noted to contain small foci of RCC. In follow-up, he has no evidence of sinonasal or thyroid recurrence after 18 months (- Fig. 1). His SNOT-22 score at his most recent visit was persistently improved to 7 , within the normal range (-Table $\mathbf{1}$ ).

\section{Case 2}

A 67-year-old white man presented with several weeks of worsening intermittent epistaxis and nasal obstruction. His SNOT-22 score at the time of initial evaluation was 23 . He had a history of T3bN2 RCC and underwent radical nephrectomy with retroperitoneal lymph node dissection 3 years prior to presentation. He developed several subcentimeter pulmonary nodules but had not received adjuvant systemic therapy given their slow growth rate. Nasal endoscopy revealed a large submucosal mass filling the medial meatus on the right and significant submucosal bulging of the posterior septum

Table 1 Comparison of pre and postoperative Sinonasal Outcome Test scores

\begin{tabular}{|l|l|l|}
\hline Case & $\begin{array}{l}\text { Preoperative } \\
\text { SNOT-22 Score }\end{array}$ & $\begin{array}{l}\text { Postoperative } \\
\text { SNOT-22 Score }\end{array}$ \\
\hline Case 1 & 32 & 7 \\
\hline Case 2 & 23 & 5 \\
\hline
\end{tabular}




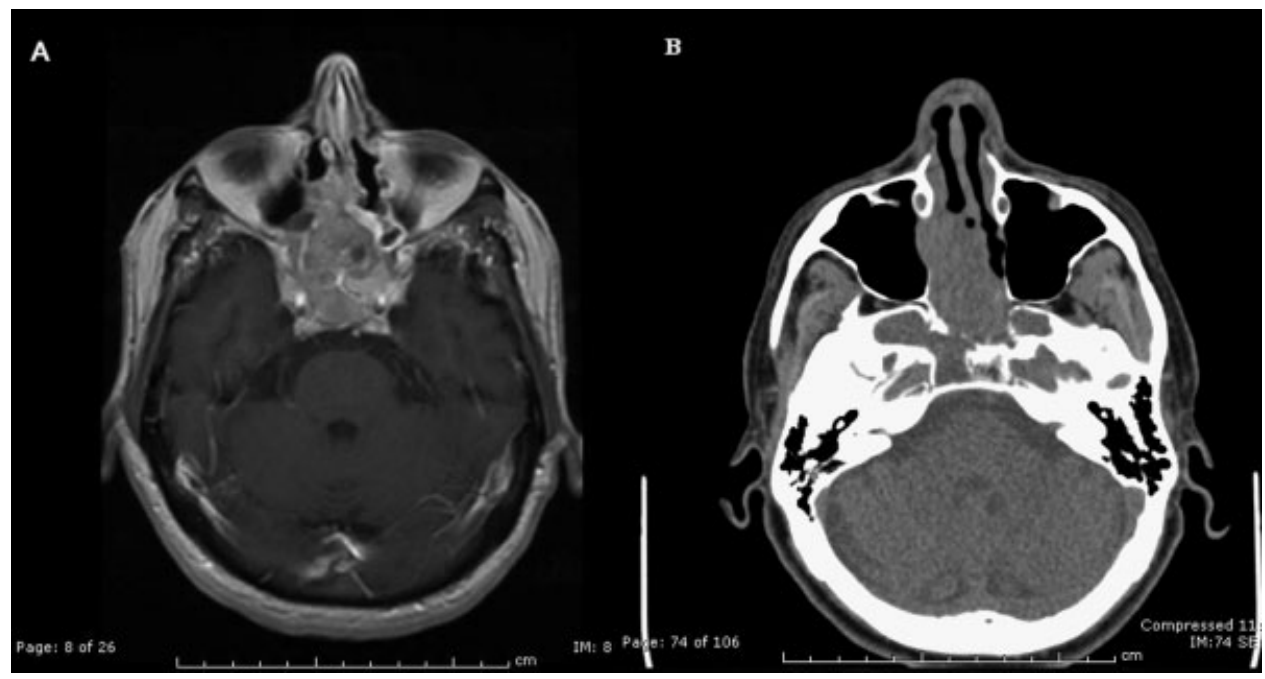

Fig. 5 (A) Axial T1 postcontrast image showing an enhancing lobulated mass centered in the posterior ethmoid air cells, extending into the sphenoid and to the clivus. (B) Axial computed tomography without contrast showing bony erosion of the right clivus.

on both sides. An outside biopsy confirmed the diagnosis of metastatic RCC.

MRI revealed a bulky $4 \times 3 \times 4 \mathrm{~cm}$ mass within the right anterior ethmoid cavity, bilateral posterior ethmoid air cells, and nasal cavity with destruction of the posterior septum, the anterior intersphenoid septum, and clivus, as well as extension of the mass into the sphenoid sinus on both sides. (-Fig. 5) There was no radiologic evidence of intracranial extension. Preoperative embolization of the mass was performed and the majority of the blood supply was from the distal branches of the internal maxillary artery bilaterally (-Fig. 6). Endoscopic resection was performed the following day. Gross tumor resection was accomplished, but the tumor extended into the clivus and was likely to have microscopic disease present in the marrow space beyond the area of clival resection. The patient tolerated the procedure well without complication and was discharged the following day. He has just completed adjuvant radiation therapy. At 3-month follow-up, his SNOT-22 score had decreased to 5.

\section{Discussion}

RCC remains a relatively rare tumor, accounting for just over $2 \%$ of all malignant disease. ${ }^{1}$ In the United States, an estimated 58,000 men and women are newly diagnosed each year, at a ratio of 1.5 to 1 , respectively. ${ }^{12}$ Metastatic disease in RCC most frequently presents in the lungs (74\%), bone (42\%), or liver $(41 \%){ }^{13}$ Fifteen percent of patients with RCC experience metastatic disease to the head and neck at or after the time of initial diagnosis. ${ }^{2}$ The most common location above the clavicle is the thyroid bed, with the nose and paranasal sinuses the second most frequent site. ${ }^{6}$ Because the classic triad of flank pain, hematuria, and a palpable abdominal mass occurs in only $10 \%$ of RCC patients, ${ }^{14}$ clinicians seeing patients

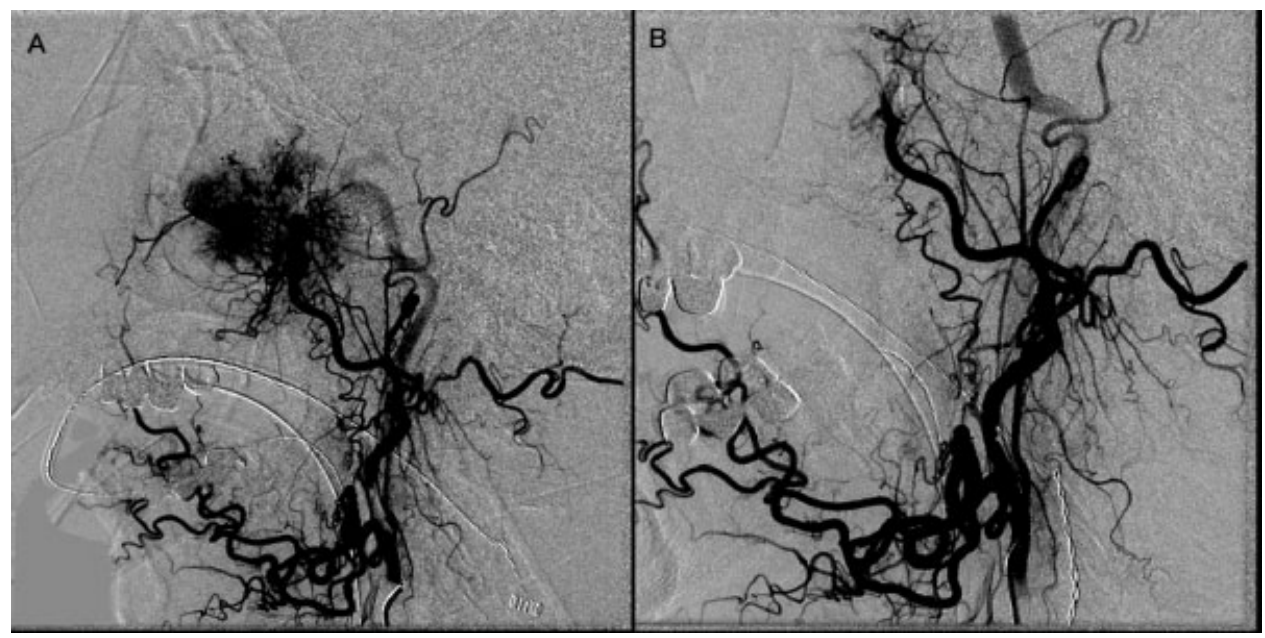

Fig. 6 (A) Right external carotid angiogram showing dense vascular contribution from the internal maxillary artery. (B) Following embolization of the internal maxillary artery. 
with symptoms of metastatic disease must maintain a high index of suspicion, even in the absence of a history of primary RCC. Overall 5-year survival rates in the United States have increased from $63.7 \%$ in 1988 to $73.9 \%$ in 2002 ; however, this may be due to a lead time bias due to earlier detection of small localized tumors incidentally noted on abdominal imaging studies. ${ }^{12}$ When reviewing patients with metastatic disease, their 5-year survival remained relatively dismal at $11.9 \%$.

Metastatic disease in RCC is thought to occur via hematogenous spread, with two possible mechanisms to the head and neck region. One is via inferior vena cava spread to the lungs, where small deposits may reside but may not yet be visible on chest radiograph. These cells may then travel to the head and neck via the carotid arterial system. This theory suggests that patients with metastatic disease in the head and neck already have widely disseminated RCC. A second possibility outlined by Nahum and Baily in 1963 is retrograde movement of tumor cells up the Batson sacral venous plexus to the head and neck, bypassing the lungs and resulting in seeding of the paranasal sinuses and thyroid as isolated metastatic sites. ${ }^{15}$ Unfortunately, the clinical behavior of RCC is frequently noted to be unpredictable in its location and timing of metastasis. ${ }^{10}$ In our series, one patient with a T1 tumor developed an isolated paranasal sinus metastasis followed by micrometastasis in the thyroid bed more than 10 years after initial diagnosis. Our second patient developed pulmonary metastases followed by a sinonasal metastasis 3 years after initial diagnosis.

Because patients may present at a significant delay from their primary treatment, diagnosis can be a challenge. Nasal obstruction and facial pain are common in many sinonasal tumors, but recurrent profuse epistaxis appears to be specific to hemangiomas and certain malignancies, including RCC and melanoma. ${ }^{8}$ In a series of six patients with metastatic RCC to the nose and paranasal sinuses, two patients who underwent biopsy had profuse bleeding that necessitated external carotid artery ligation. ${ }^{10}$ Spontaneous epistaxis from these lesions can result in anemia and require recurrent transfusions, ${ }^{8}$ and authors in one report argue that even in the setting of multiple sinonasal metastases, endoscopic resection should be performed with the primary purpose of limiting recurrent, massive epistaxis and improving quality of life. ${ }^{16}$

In general, work-up for a sinonasal mass should include endoscopic examination, followed by a CT scan with contrast, prior to considering biopsy. This is the imaging test of choice for its ability to delineate vascularity and skull base involvement. Typically, metastatic RCC lacks tumor calcification and is brightly enhancing on CT with contrast, similar to the CT appearance of primary renal disease. ${ }^{17}$

With superior visualization and ability to control intraoperative epistaxis, endoscopic biopsy has now become the standard of care for diagnosis of a sinonasal mass. ${ }^{18}$ Pathologic specimens typically demonstrate clear cytoplasm and show cells arranged in nests and cords with prominent vascular lakes (-Fig. 4). The histologic diagnosis may be supported by RCC antigen immunostain.

In the largest case series in the literature of patients with RCC metastatic to the nose and paranasal sinuses, Simo et al discuss six patients, ${ }^{10}$ all treated with radiotherapy following biopsy. They argue that although RCC has been thought of as a radioresistant tumor, this is primarily because of the inability to obtain high doses of radiation in the retroperitoneum, and that the anatomy of the nose and paranasal sinuses would allow for an effective dose of radiation to treat the disease. Thirty percent of their patients did have a complete response, whereas 50\% had a partial response and one had no response (stable disease). Patients did require periodic nasal decrusting following external beam radiation, but the authors did not elaborate on the effect of treatment on quality of life. At 26 months, four of their patients had died and two remained alive. The majority of the patients who died had advanced metastatic disease in the lung or bone as well.

Two previous reports describe the successful endoscopic resection of localized disease in the paranasal sinuses, noting survival rates greater than 5 years after surgery. ${ }^{2,8}$ In the past, radiation therapy alone has been the treatment of choice for metastatic disease. Our case series has demonstrated that for isolated lesions that impact on patient quality of life, endoscopic resection can be effective and safe, carrying minimal morbidity and greatly improving sinonasal complaints. Although epidemiologic data suggest that 5 -year survival for patients with metastatic disease is low, ${ }^{12}$ there is a real opportunity to improve patient symptoms through management of sinonasal metastases. Local control of head and neck metastases may allow patients improved quality of life while receiving systemic adjuvant therapy for more widely disseminated disease. Adjuvant treatment now includes anti-vascular endothelial growth factor (VEGF) and mTOR pathway inhibitors, which have been shown to improve progressionfree survival in advanced RCC. ${ }^{19}$ These agents are now being added to our treatment plans where appropriate.

At our institution, preoperative embolization and endoscopic resection followed by proton beam radiation is the treatment algorithm for resectable disease. Embolization provides a superior operative field and visualization while limiting blood loss, and has been shown to be extremely safe in the head and neck. ${ }^{20}$ Even when feeding vessels arise from the internal carotid system, the preoperative angiogram and external system embolization can greatly help with surgical planning. Our limited series of patients had minimal blood loss and no significant morbidity, and they were discharged the day following surgery. Their sinonasal symptoms mirror that of a healthy population on the SNOT-22, ${ }^{11}$ and in our short period of follow-up, there has been no evidence of local disease recurrence.

\section{Conclusion}

RCC is a relatively rare tumor with unpredictable metastatic potential. Sinonasal metastases may be the initial presentation of this disease. Definitive diagnosis depends on histopathology, and endoscopic biopsy can be used for this purpose. If metastatic RCC is suspected, preoperative preparation for blood loss is essential given the significant vascularity of this tumor. Metastatic sinonasal RCC has been traditionally treated with radiation therapy. Our case series demonstrates that patients with metastatic RCC to the sinonasal cavity can 
be safely and successfully treated with preoperative embolization followed by endoscopic resection. Our patients had no significant blood loss and were discharged the day following surgery. They also had a significant improvement in sinonasal symptoms and quality of life. The treatment algorithm at our institution currently involves endoscopic surgical resection for lesions that can be removed with minimal morbidity, followed by radiation therapy.

\section{Acknowledgments}

We have no disclosures.

\section{Presentations}

This paper was presented at the North American Skull Base Society Meeting in Las Vegas, Nevada in February 2012.

\section{References}

1 Ferlay J, Shin H, Bray F. GLOBOCAN 2008: cancer incidence and mortality worldwide. Lyon (France): International Agency for Research on Cancer; 2010

2 Gottlieb MD, Roland JT Jr. Paradoxical spread of renal cell carcinoma to the head and neck. Laryngoscope 1998;108(9):1301-1305

3 Donaldson JC, Slease RB, DuFour DR, Saltzman AR. Metastatic renal cell carcinoma 24 years after nephrectomy. JAMA 1976;236(8): 950-951

4 Azam F, Abubakerr M, Gollins S. Tongue metastasis as an initial presentation of renal cell carcinoma: a case report and literature review. J Med Case Reports 2008;2:249

5 Bhatia S, Ng S, Hodder SC. Metastatic cutaneous head and neck renal cell carcinoma with no known primary: case report. Br J Oral Maxillofac Surg 2010;48(3):214-215
6 Nixon IJ, Whitcher M, Glick J, et al. Surgical management of metastases to the thyroid gland. Ann Surg Oncol 2011;18(3): 800-804

7 Kathuria S, Molnar Z, Reyes CV. Renal cell carcinoma presenting as epistaxis. J Surg Oncol 1980;14(2):153-157

8 Lee HM, Kang HJ, Lee SH. Metastatic renal cell carcinoma presenting as epistaxis. Eur Arch Otorhinolaryngol 2005;262(1):69-71

9 Ziari M, Shen S, Amato RJ, Teh BS. Metastatic renal cell carcinoma to the nose and ethmoid sinus. Urology 2006;67(1):199

10 Simo R, Sykes AJ, Hargreaves SP, et al. Metastatic renal cell carcinoma to the nose and paranasal sinuses. Head Neck 2000;22(7):722-727

11 Gillett S, Hopkins C, Slack R, Browne JP. A pilot study of the SNOT 22 score in adults with no sinonasal disease. Clin Otolaryngol 2009; 34(5):467-469

12 Cho E, Adami HO, Lindblad P. Epidemiology of renal cell cancer. Hematol Oncol Clin North Am 2011;25(4):651-665

13 Saitoh H. Distant metastasis of renal adenocarcinoma. Cancer 1981;48(6):1487-1491

14 Skinner DG, Colvin RB, Vermillion CD, Pfister RC, Leadbetter WF Diagnosis and management of renal cell carcinoma. A clinical and pathologic study of 309 cases. Cancer 1971;28(5):1165-1177

15 Nahum AM, Bailey BJ. Malignant Tumors Metastatic to the Paranasal Sinuses: Case Report and Review of the Literature. Laryngoscope 1963;73:942-953

16 Terada N, Hiruma K, Suzuki M, Numata T, Konno A. Metastasis of renal cell cancer to the ethmoid sinus. Acta Otolaryngol Suppl 1998;537:82-86

17 Som PM, Norton KI, Shugar JM, et al. Metastatic hypernephroma to the head and neck. AJNR Am J Neuroradiol 1987;8(6): 1103-1106

18 Homer JJ, Jones NS, Bradley PJ. The role of endoscopy in the management of nasal neoplasia. Am J Rhinol 1997;11(1):41-47

19 Coppin C, Kollmannsberger C, Le L, Porzsolt F, Wilt TJ. Targeted therapy for advanced renal cell cancer (RCC): a Cochrane systematic review of published randomised trials. BJU Int 2011;108(10): 1556-1563

20 Cooke D, Ghodke B, Natarajan SK, Hallam D. Embolization in the head and neck. Semin Intervent Radiol 2008;25(3):293-309 